\title{
Correlated Set Coordination in Fault Tolerant Message Logging Protocols
}

\author{
Aurelien Bouteiller ${ }^{1}$, Thomas Herault ${ }^{1}$, George Bosilca ${ }^{1}$, \\ and Jack J. Dongarra ${ }^{1,2}$ \\ 1 Innovative Computing Laboratory, \\ The University of Tennessee \\ 2 Oak Ridge National Laboratory \\ \{bouteill, herault, bosilca, dongarra\}@eecs.utk. edu
}

\begin{abstract}
Based on our current expectation for the exascale systems, composed of hundred of thousands of many-core nodes, the mean time between failures will become small, even under the most optimistic assumptions. One of the most scalable checkpoint restart techniques, the message logging approach, is the most challenged when the number of cores per node increases, due to the high overhead of saving the message payload. Fortunately, for two processes on the same node, the failure probability is correlated, meaning that coordinated recovery is free. In this paper, we propose an intermediate approach that uses coordination between correlated processes, but retains the scalability advantage of message logging between independent ones. The algorithm still belongs to the family of event logging protocols, but eliminates the need for costly payload logging between coordinated processes.
\end{abstract}

\section{Introduction}

High Performance Computing, as observed by the Top 500 ranking 1 , has exhibited a constant progression of the computing power by a factor of two every 18 months for the last 15 years. Following this trend, the exaflops milestone should be reached as soon as 2019. The International Exascale Software Project (IESP) 7] proposes an outline of the characteristics of an exascale machine, based on the foreseeable limits of the hardware and maintenance costs. A machine in this performance range is expected to be built from gigahertz processing cores, with thousands of cores per computing node (up to $10^{12}$ flops per node), thus requiring millions of computing nodes to reach the exascale. Software will face the challenges of complex hierarchies and unprecedented levels of parallelism.

One of the major concerns is reliability. If we consider that failures of computing nodes are independent, the reliability probability of the whole system (i.e. the probability that all components will be up and running during the next time unit) is the product of the reliability probability of each of the components. A conservative assumption of a ten years mean time to failure translates into a

\footnotetext{
1 http://www.top500.org/
} 
probability of 0.99998 that a node will still be running in the next hour. If the system consists of a million of nodes, the probability that at least one unit will be subject to a failure during the next hour jumps to $1-0.99998^{10^{6}}>0.99998$. This probability being disruptively close to 1 , one can conclude that many computing nodes will inevitably fail during the execution of an exascale application.

Automatic fault tolerant algorithms, which can be provided either by the operating system or the middleware, remove some of the complexity in the development of applications by masking failures and the ensuing recovery process. The most common approaches to automatic fault tolerance are replication, which consumes a high number of computing resources, and rollback recovery. Rollback recovery stores system-level checkpoints of the processes, enabling rollback to a saved state when failures happen. Consistent sets of checkpoints must be computed, using either coordinated checkpointing or some variant of uncoordinated checkpointing with message logging (for brevity, in this article, we use indifferently message logging or uncoordinated checkpointing). Coordinated checkpointing minimizes the overhead of failure-free operations, at the expense of a costly recovery procedure involving the rollback of all processes. Conversely, message logging requires every communication to be tracked to ensure consistency, but its uncoordinated recovery procedure demonstrates unparalleled efficiency in failure prone environments.

Although the low mean time to failure of exascale machines calls for preferring an uncoordinated checkpoint approach, the overhead on communication of message logging is bound to increase with the advent of many-core nodes. Uncoordinated checkpointing has been designed with the idea that failures are mostly independent, which is not the case in many-core systems where multiple cores crash when the node is struck by a failure. Not only do simultaneous failures negate the advantage of uncoordinated recovery, but the logging of messages between cores is also a major performance issue. All interactions between two uncoordinated processes have to be logged, and a copy of the transaction must be kept for future replay. Since making a copy has the same cost as doing the transaction itself (as the processes are on the same node we consider the cost of communications equal to the cost of memory copies), the overhead is unacceptable. It is disconcerting that the most resilient fault tolerant method is also the most bound to suffer, in terms of performance, on expected future systems.

In this paper, we consider the case of correlated failures: we say that two processes are correlated or co-dependent if they are likely to be subject to a simultaneous failure. We propose a hybrid approach between coordinated and non coordinated checkpointing, that prevents the overhead of keeping message copies for communications between correlated processes, but retains the more scalable uncoordinated recovery of message logging for processes whose failure probability is independent. The coordination protocol we present is a split protocol, which takes into account the fragmentation of messages, to avoid long waiting cycles, while still implementing a transactional semantic for whole messages. 


\section{Rollback Recovery Background}

\subsection{Execution Model}

Events and States: Each computational or communication step of a process is an event. An execution is an alternate sequence of events and process states, with the effect of an event on the preceding state leading the process to the new state. As the system is basically asynchronous, there is no direct time relationship between events occurring on different processes. However, Lamport defines a causal partial ordering between events with the happened before relationship [14].

Events can be classified into two categories. An event is deterministic when, from the current state, all executions lead to the same outcome state. On the contrary, if in different executions, the same event happening on a particular state can result in several different outcome states, then it is nondeterministic. Examples of nondeterministic events are message receptions, which depend on external influences like network jitter.

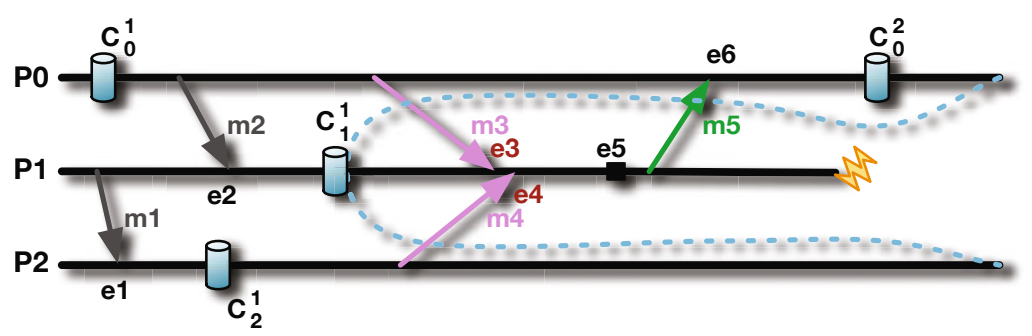

Fig. 1. Recovery line based on rollback recovery of a failed process

Recovery Line: Rollback recovery addresses mostly fail-stop errors: a failure is the loss of the complete state and actions of a process. A checkpoint is a copy of a past state of a particular process stored on some persistent memory (remote node, disk, ...), and used to restore the process in case of failure. The recovery line is the configuration of the entire application after some processes have been reloaded from checkpoints. If the checkpoints can happen at arbitrary dates, some messages can cross the recovery line. Consider the example execution of Figure 11. When the process $P_{1}$ fails, it rolls back to checkpoint $C_{1}^{1}$. If no other process rolls back, messages $m_{3}, m_{4}, m_{5}$ are crossing the recovery line. A recovery set is the union of the saved states (checkpoint, messages, events) and a recovery line.

In-transit Messages: Messages $m_{3}$ and $m_{4}$ are crossing the recovery line from the past, they are called in-transit messages. The in-transit messages are necessary for the progression of the recovered processes, but are not available anymore, as the corresponding send operation is in the past of the recovery line. For a recovery line to form a complete recovery set, every in-transit message must be added to the recovery line. 
Orphan Messages: Message $m_{5}$ is crossing the recovery line from the future to the past; such messages are referred to as orphan messages. By following the happened-before relationship, the current state of $P_{0}$ depends on the reception of $m_{5}$; by transitivity, it also depends on events $e_{3}, e_{4}, e_{5}$ that occurred on $P_{1}$ since $C_{1}^{1}$. Since the channels are asynchronous, the reception of $m_{3}$ and $m_{4}$, from different senders, can occur in any order during re-execution, leading to a recovered state of $P_{1}$ that can diverge from the initial execution. As a result, the current state of $P_{0}$ depends on a state that $P_{1}$ might never reach after recovery. Checkpoints leading to such inconsistent states are useless and must be discarded; in the worst case, a domino effect can force all checkpoints to be discarded.

\subsection{Building a Consistent Recovery Set}

Two different strategies can be used to create consistent recovery sets. The first one is to create checkpoints at a moment in the history of the application where no orphan messages exist, usually through coordination of checkpoints. The second approach avoids coordination, but instead saves all in-transit messages to be able to replay those without rollback, and keep track of nondeterministic events, so that orphan messages can be regenerated identically. We focus our work on this second approach, deemed more scalable.

Coordinated Checkpoint: Checkpoint coordination aims at eliminating intransit and orphan messages from the recovery set. Several algorithms have been proposed to coordinate checkpoints, the most usual being the ChandyLamport algorithm [6] and the blocking coordinated checkpointing, 517], which silences the network. In these algorithms, waves of tokens are exchanged to form a recovery line that eliminates orphan messages and detects in-transit messages. Coordinated algorithms have the advantage of having almost no overhead outside of checkpointing periods, but require that every process, even if unaffected by failures, rolls back to its last checkpoint, as this is the only recovery line that is guaranteed to be consistent.

Message Logging: Message Logging is a family of algorithms that attempt to provide a consistent recovery set from checkpoints taken at independent dates. As the recovery line is arbitrary, every message is potentially in-transit or orphan. Event Logging is the mechanism used to correct the inconsistencies induced by orphan messages, and nondeterministic events, while Payload Copy is the mechanism used to keep the history of in-transit messages. While introducing some overhead on every exchanged message, this scheme can sustain a much more adverse failure pattern, which translates to better efficiency on systems where failures are frequent [15].

Event Logging: In event logging, processes are considered Piecewise deterministic: only sparse nondeterministic events occur, separating large parts of deterministic computation. Event logging suppresses future nondeterministic events 
by adding the outcome of nondeterministic events to the recovery set, so that it can be forced to a deterministic outcome (identical to the initial execution) during recovery. The network, more precisely the order of reception, is considered the unique source of nondeterminism. The relative ordering of messages from different senders $\left(e_{3}, e_{4}\right.$ in fig. 1), is the only information necessary to be logged. For a recovery set to be consistent, no unlogged nondeterministic event can precede an orphan message.

Payload Copy: When a process is recovering, it needs to replay any reception that happened between the last checkpoint and the failure. Consequently, it requires the payload of $i n$-transit messages $\left(m_{3}, m_{4}\right.$ in fig 1). Several approaches have been investigated for payload copy, the most efficient one being the senderbased copy [18. During normal operation, every outgoing message is saved in the sender's volatile memory. The surviving processes can serve past messages to recovering processes on demand, without rolling back. Unlike events, senderbased data do not require stable or synchronous storage (although this data is also part of the checkpoint). Should a process holding useful sender-based data crash, the recovery procedure of this process replays every outgoing send and thus rebuilds the missing messages.

\section{Group-Coordinated Message Logging}

\subsection{Shared Memory and Message Logging}

Problem Statement: In uncoordinated checkpoint schemes, the ordering between checkpoint and message events is arbitrary. As a consequence, every message is potentially in-transit, and must be copied. Although the cost of the sender-based mechanism involved to perform this necessary copy is not negligible, the cost of a memory copy is often one order of magnitude lower than the cost of the network transfer. Furthermore, the copy and the network operation can overlap. As a result, proper optimization greatly mitigates the performance penalty suffered by network communications (typically to less than 10\%, 23] ). One can hope that future engineering advances will further reduce this overhead.

Unlike a network communication, a shared memory communication is a strongly memory-bound operation. In the worst case, memory copy induced by message logging doubles the volume of memory transfers. Because it competes for the same scarce resource - memory bandwidth - the cost of this extra copy cannot be overlapped, hence the time to send a message is irremediably doubled.

A message is in-transit (and needs to be copied) if it crosses the recovery line from the past to the future. The emission and reception dates of messages are beyond the control of the fault tolerant algorithm: one could delay the emission or reception dates to match some arbitrary ordering with checkpoint events, but these delays would obviously defeat the goal of improving communication performance. The only events that the fault tolerant algorithm can alter, to enforce an ordering between message events and checkpoint events, are checkpoint dates. Said otherwise, the only way to suppress in-transit messages is to synchronize checkpoints. 
Correlated Failures: Fortunately, although many-core machines put a strain on message logging performance, a new opportunity opens, thanks to the side effect that failures do not have an independent probability on such an environment. All the processes hosted by a single many-core node are prone to fail simultaneously: they are located on the same piece of silicon, share the same memory bus, network interface, cooling fans, power supplies, operating system, and are subject to the same physical interferences (rays, heat, vibrations, ...). One of the motivating properties of message logging is that it tolerates a large number of independent failures very well. If failures are correlated, the fault tolerant algorithm can be more synchronous without decreasing its effective efficiency.

The leading idea of our approach is to propose a partially coordinated fault tolerant algorithm, that retains message logging between sets of processes experiencing independent failure probability, but synchronize the checkpoints of processes that have a strong probability of simultaneous failures, what we call a correlated set. It leverages the correlated failures property to avoid message copies that have a high chance of being useless.

\subsection{Correlated Set Coordinated Message Logging}

Whenever a process of a correlated set needs to take a checkpoint, it forces a synchronization with all other processes of the set. If a failure hits a process, all processes of that set have to roll back to their last checkpoint (see the recovery line in example execution depicted in figure 2). Considering a particular correlated set, every message can be categorized as either ingoing $\left(m_{1}, m_{2}\right)$, outgoing $\left(m_{5}\right)$, or internal $\left(m_{3}, m_{4}\right)$. Between sets, no coordination is enforced. A process failing in another correlated set does not trigger a rollback, but messages between sets have no guaranteed properties with respect to the recovery line, and can still be orphan or in-transit. Therefore, regular message logging, including payload copy and event logging must continue for outgoing and ingoing messages.

As checkpoints are coordinated, all orphan and in-transit messages are eliminated between processes of the correlated set. However, as the total recovery

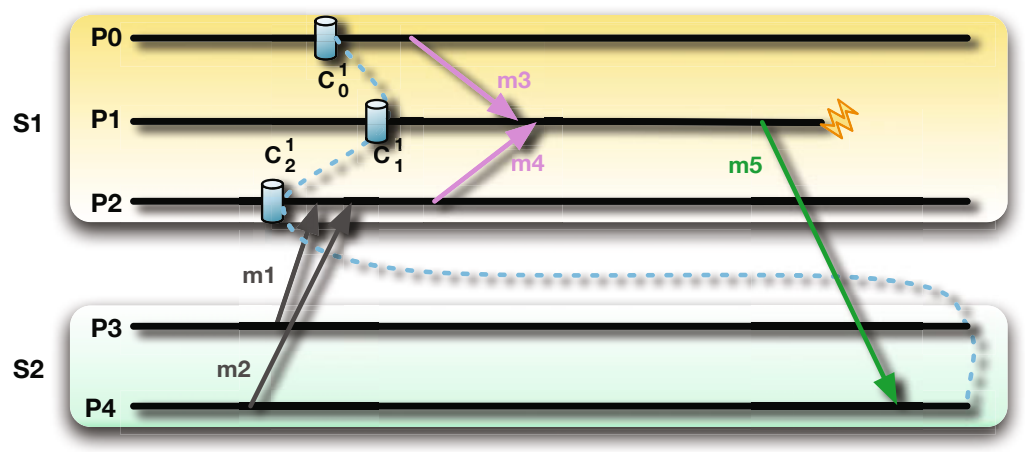

Fig. 2. An execution of the Correlated Set Coordinated Message Logging Algorithm 
set does contain in-transit and orphan messages, the consistency proof of coordinated checkpoint does not hold for the recovery set formed by the union of the coordinated sets. In an uncoordinated protocol, a recovery set is consistent if all in-transit messages are available, and no orphan message depends on the outcome of a non-deterministic event. In the next paragraphs, we demonstrate that payload copy can be disabled for internal messages, but that event logging must apply to all types of messages.

Intra-set Payload Copy: By the direct application of the coordination algorithm, no message is in-transit between any pair of synchronized processes at the time of checkpoint (in the case of the Chandy/Lamport algorithm, occasional in-transit messages are integrated inside the checkpoint, hence they are not in-transit anymore). Because an internal message cannot be in-transit, it is never sent before the recovery line and received after. Therefore, the payload copy mechanism, used to recover past sent messages during the recovery phase, is unnecessary for internal messages.

\section{Intra-set Event Logging:}

Theorem 1. In a fault tolerant protocol creating recovery sets with at least two distinct correlated sets, if the nondeterministic outcome of any internal messages preceding an outgoing message is omitted from the recovery set, there exists an execution that reaches an inconsistent state.

Outgoing messages are crossing a non-coordinated portion of the recovery line, hence the execution follows an arbitrary ordering between checkpoint events and message events. Therefore, for any outgoing message there is an execution in which it is orphan. Consider the case of the execution depicted in figure 2 , In this execution, the message $m_{5}$, between the sets $S_{1}$ and $S_{2}$ is orphan in the recovery line produced by a rollback of the processes of $S_{1}$.

Let's suppose that Event logging of internal messages is unnecessary for building a consistent recovery set. The order between the internal receptions and any other reception of the same process on another channel is nondeterministic. By transitivity of the Lamport relationship, this nondeterminism is propagated to the dependent outgoing message. Because an execution in which this outgoing message is orphan exists, the recovery line in this execution is inconsistent. The receptions of messages $m_{3}, m_{4}$ are an example: the nondeterministic outcome created by the unknown ordering of messages in asynchronous channels is propagated to $P_{4}$ through $m_{5}$. The state of the correlated set $S_{2}$ depends on future nondeterministic events of the correlated set $S_{1}$, therefore the recovery set is inconsistent. One can also remark that the same proof holds for ingoing messages (as illustrated by $m_{1}$ and $m_{2}$ ).

As a consequence of this theorem, it is necessary to log all message receptions, even if the emitter is located in the same correlated set as the receiver. Only the payload of this message can be spared. 


\subsection{Implementation}

We have implemented the correlated set coordinated message logging algorithm inside the Open MPI library. Open MPI [9] is one of the leading Message Passing Interface standard implementations [19. In Open MPI, the PML-V framework enables researchers to express their fault tolerant policies. The Vprotocol Pessimist is such an implementation of a pessimistic message logging protocol [3]. In order to evaluate the performance of our new approach, we have extended this fault tolerant component with the capabilities listed below.

Construction of the Correlated Set, Based on Hardware Proximity: Open MPI enables the end user to select a very precise mapping of his application on the physical resources, up to pinning a particular MPI rank to a particular core. As a consequence, the Open MPI's runtime instantiates a process map detailing node hierarchies and ranks allocations. The detection of correlated sets parses this map and extracts the groups of processes hosted on the same node.

Internal Messages Detection: In Open MPI, the couple formed by the rank and the communicator is translated into a list of endpoints, each one representing a channel to the destination (eth0, ib0, shared memory, ...). During the construction of the correlated set, all endpoints pertaining to a correlated process are marked. When the fault tolerant protocol considers making a sender-based copy, the endpoint is checked to determine if the message payload has to be copied.

Checkpoint Coordination in a Correlated Set: The general idea of a network-silence based coordination is simple: processes send a marker in their communication channels to notify other processes that no other message will be sent before the end of the phase. When all output channels and input channels have been notified, the network is silenced, and the processes can start communicating again. However, MPI communications do not exactly match the theoretical model, which assumes message emissions or receptions are atomic events. In practice, an MPI message is split into several distinct events. The most important include the emission of the first fragment (also called eager fragment), the matching of an incoming fragment with a receive request, and the delivery of the last fragment. Most of those events are unordered, in particular, a fragment can overtake another fragment, even from the same message (especially with channel bonding). Fortunately, because the MPI matching has to be FIFO, in Open MPI, eager fragments are FIFO, an advantageous property that our algorithm leverages. Our coordination algorithm has three phases: it silences eager fragments so that all posted sends are matched; it completes any matched receives; it checkpoints processes in the correlated set.

Eager silence: When a process enters the checkpoint synchronization, it sends a token to all correlated opened endpoints. Any send targeting a correlated endpoint, if posted afterwards, is stalled upon completion of the algorithm. When 
a process not yet synchronizing receives a token, it enters the synchronization immediately. The eager silence phase is complete for a process when it has received a token from every opened endpoint. Because no new message can inject an eager fragment after the token, and eager fragments are FIFO, at the end of this phase, all posted sends of processes in the correlated set have been matched.

Rendez-vous Silence: Unlike eager fragments, the remainder fragments of a message can come in any order. Instead of a complex non-FIFO token algorithm, the property that any fragment left in the channel belongs to an already matched message can be leveraged to drain remaining fragments. In the rendez-vous silence phase, every receive request is considered in turn. If a request has matched an eager fragment from a process of the correlated set, the progress engine of Open MPI is called repeatedly until it is detected that this particular request completed. When all such requests have completed, all fragments of internal messages to this process have been drained.

Checkpoint phase: When a process has locally silenced its internal inbound channels, it enters a local barrier. After the barrier, all channels are guaranteed to be empty. Each process then takes a checkpoint. A second barrier denotes that all processes finished checkpointing and that subsequent sends can be resumed.

\section{Experimental Evaluation}

\subsection{Experimental Conditions}

The Pluto platform features 48 cores, and is our main testbed for large shared memory performance evaluations. Pluto is based on four 12-core AMD opteron 6172 processors with $128 \mathrm{~GB}$ of memory. The operating system is Red Hat 4.1 .2 with the Linux 2.6.35.7 kernel. Despite the NUMA hierarchies, in this machine, the bandwidth is almost equal between all pairs of cores. The Dancer cluster is an 8 node cluster, where each node has two quad-core Intel Xeon E5520 CPUs, with $4 \mathrm{~GB}$ of memory. The operating system is Caos NSA with the 2.6.32.6 Linux kernel. Nodes are connected through an Infiniband 20G network.

All protocols are implemented in Open MPI devel r20284. Vanilla Open MPI means that no fault tolerant protocol is enabled, regular message logging means that the pessimistic algorithm is used, and coordinated message logging denotes that cores of the same node belong to a correlated set. The evaluation includes synthetic benchmarks, such as NetPIPE 3.7 and IMB 3.3, and application benchmarks, such as the NAS 3.3 and HPL (with MKL BLAS10.2). The different benchmarks of the NAS suite accept a constrained number of processes (some expect a square number of processes, others a power of two). In all cases, we ran the largest possible experiment, for a given benchmark and a given parallel machine. 


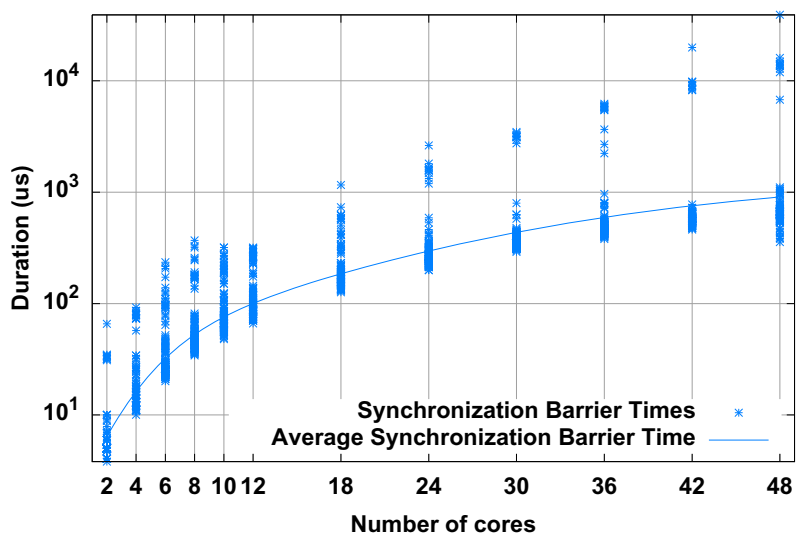

Fig. 3. Time to synchronize a correlated set (Pluto platform, log/log scale)

\subsection{Shared Memory Performance}

Coordination Cost: The cost of coordinating a growing number of cores is presented in the figure 3. The first token exchange is a complete all-to-all, that cannot rely on a spanning tree algorithm. Although, all other synchronizations are simple barriers, the token exchange dominates the execution time, which grows quadratically with the number of processes. Note, however, that this synchronization happens only during a checkpoint, and that its average cost is comparable to sending a 10KB message. Clearly, the cost of transmitting a checkpoint to the I/O nodes overshadows the cost of this synchronization.

Ping Pong: Figure 4 presents the results of the NetPIPE benchmark on shared memory with a logarithmic scale. Processes are pinned to two cores sharing an L2 cache, a worst case scenario for regular message logging. The maximum bandwidth reaches $53 \mathrm{~Gb} / \mathrm{s}$, because communication cost is mostly related to accessing the L2 cache. The sender-based algorithm decreases the bandwidth to $11 \mathrm{~Gb} / \mathrm{s}$, because it copies data to a buffer that is never in the cache. When the coordination algorithm allows for disabling the sender-based mechanism, event logging obtains the same bandwidth as the non fault tolerant execution.

NAS Benchmarks: Figure 5 presents the performance of the NAS benchmarks on the shared memory Pluto platform. BT and SP run on 36 cores, all others run on 32. One can see that avoiding payload copy enables the coordinated message logging algorithm to experience at most a $7 \%$ slowdown, and often no overhead, while the regular message logging suffers from up to $17 \%$ slowdown. 


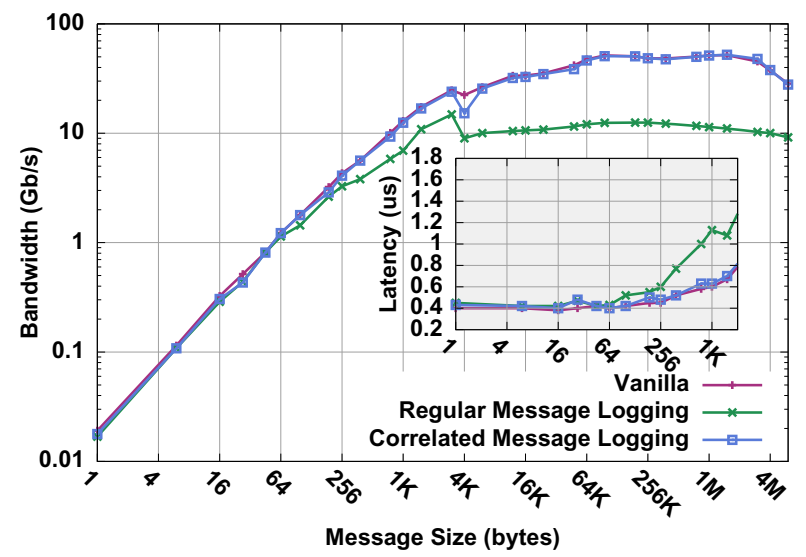

Fig. 4. Ping pong performance (Dancer node, shared memory, $\log / \log$ scale)

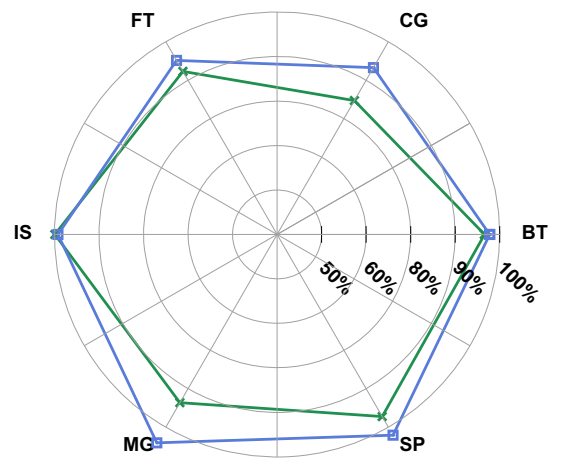

Perf. Regular Message Logging / Perf. Vanilla Perf. Coordinated Message Logging / Perf. Vanilla

Fig. 5. NAS performance (Pluto platform, shared memory, 32/36 cores)

\subsection{Cluster of Multicore Performance}

Figure 6 presents the performance of the HPL benchmark on the Dancer cluster, with a one process per core deployment. For small matrix sizes, the behavior is similar between the three MPI versions. However, for slightly larger matrix sizes, the performance of regular message logging suffers. Conversely the coordinated message logging algorithm performs better, and only slightly slower than the non fault tolerant MPI, regardless of the problem size.

On the Dancer cluster, the available $500 \mathrm{MB}$ of memory per core is a strong limitation. In this memory envelope, the maximum computable problem size on this cluster is $\mathrm{N}=28260$. The extra memory consumed by payload copy limits the maximum problem size to only $\mathrm{N}=12420$ for regular message logging, while the 


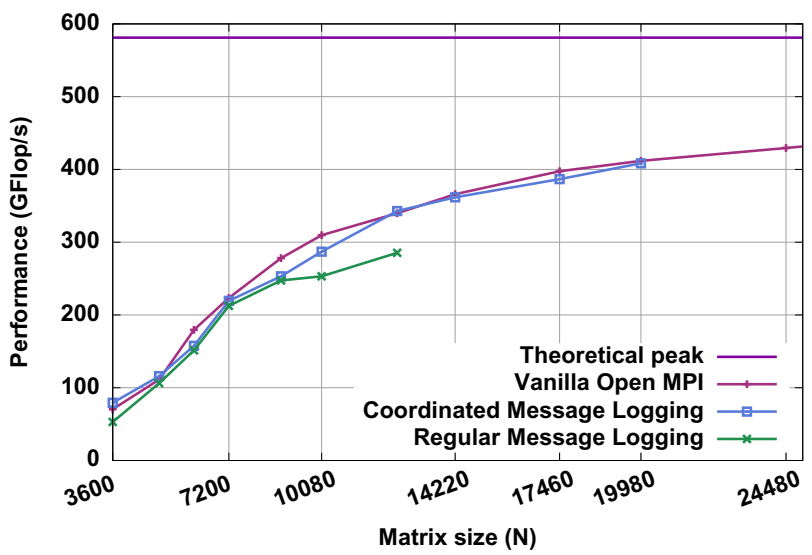

Fig. 6. HPL cluster performance (Dancer cluster, IB20G, 8 nodes, 64 cores)

reduction on the amount of logged messages enables the coordinated message logging approach to compute problems as large as $\mathrm{N}=19980$. Not only does partial coordination of the message logging algorithm increase communication performance, it also decreases memory consumption.

\section{Related Works}

Recent advances in message logging have decreased the cost of event logging 3 . As a consequence, more than the logging scheme adopted, the prominent source of overhead in message logging is the copy of message payload caused by intransit messages [4. While attempts at decreasing the cost of payload copy have been successful to some extent [2, these optimizations are hopeless at improving shared memory communication speed. Our approach circumvents this limitation by completely eliminating the need for copies inside many-core processors.

Communication Induced Checkpoint (CIC) [12 is another approach that aims at constructing a consistent recovery set without coordination. The CIC algorithm maintains the dependency graph of events and checkpoints to compute $Z$-paths as the execution progresses. Forced checkpoints are taken whenever a Z-path would become a consistency breaking Z-cycle. This approach has several drawbacks: it adds piggyback to messages, and is notably not scalable because the number of forced checkpoints grows uncontrollably [1].

Group coordinated checkpoint have been proposed in MVAPICH2 10] to solve I/O storming issues in coordinated checkpointing. In this paper, the group coordination refers to a particular scheduling of the checkpoint traffic, intended to avoid overwhelming the I/O network. Unlike our approach, which is partially uncoordinated, this algorithm builds a completely coordinated recovery set.

In [11, Ho, Wang and Lau propose a group-based approach that combines coordinated and uncoordinated checkpointing, similar to the technique we use in this paper, to reduce the cost of message logging in uncoordinated checkpointing. Their 
work, however, focuses on communication patterns of the application, to reduce the amount of message logging. Similarly, in the context of Charm ++ [13], and AMPI [16], Meneses, Mendes and Kalé have proposed in [8] a team-based approach to reduce the overhead of message logging. The Charm ++ model advocates a high level of oversubscription, with a ratio of user-level thread per core much larger than one. In their work, teams are of fixed, predetermined sizes. The paper does not explicitly explain how teams are built, but an emphasis on communication patterns seems preferred. In contrast, our work takes advantage of hardware properties of the computing resources, proposing to build correlated groups based on likeliness of failures, and relative efficiency of the communication medium.

\section{Concluding Remarks}

In this paper, we proposed a novel approach combining the best features of coordinated and uncoordinated checkpointing. The resulting fault tolerant protocol, belonging to the event logging protocol family, spares the payload logging for messages belonging to a correlated set, but retains uncoordinated recovery scalability. The benefit on shared memory point-to-point performance is significant, which translates into an observable improvement of many application types. Even though inter-node communications are not modified by this approach, the shared memory speedup translates into a reduced overhead on cluster of multicore type platforms. Last, the memory required to hold message payload is greatly reduced; our algorithm provides a flexible control of the tradeoff between synchronization and memory consumption. Overall, this work greatly improves the applicability of message logging in the context of distributed systems based on a large number of many-core nodes.

Acknowledgement. This work was partially supported by the DOE Cooperative Agreement DE-FC02-06ER25748, and the INRIA-Illinois Joint Laboratory for Petascale Computing and the ANR RESCUE project.

\section{References}

1. Alvisi, L., Elnozahy, E., Rao, S., Husain, S.A., Mel, A.D.: An analysis of communication induced checkpointing. In: 29th Symposium on Fault-Tolerant Computing (FTCS 1999). IEEE CS Press, Los Alamitos (1999)

2. Bosilca, G., Bouteiller, A., Herault, T., Lemarinier, P., Dongarra, J.J.: Dodging the cost of unavoidable memory copies in message logging protocols. In: Keller, R., Gabriel, E., Resch, M., Dongarra, J. (eds.) EuroMPI 2010. LNCS, vol. 6305, pp. 189-197. Springer, Heidelberg (2010)

3. Bouteiller, A., Bosilca, G., Dongarra, J.: Redesigning the message logging model for high performance. In: ISC 2008, Wiley, Dresden (June 2008) (p. to appear)

4. Bouteiller, A., Ropars, T., Bosilca, G., Morin, C., Dongarra, J.: Reasons to be pessimist or optimist for failure recovery in high performance clusters. In: IEEE (ed.) Proceedings of the 2009 IEEE Cluster Conference (September 2009) 
5. Buntinas, D., Coti, C., Herault, T., Lemarinier, P., Pilard, L., Rezmerita, A., Rodriguez, E., Cappello, F.: Blocking vs. non-blocking coordinated checkpointing for large-scale fault tolerant MPI protocols. Future Generation Computer Systems 24(1), 73-84 (2008), http://www.sciencedirect.com/science/article/B6V06-4N2KT6H-1/2/ 00e790651475028977cc3031d9ea3980

6. Chandy, K.M., Lamport, L.: Distributed snapshots: Determining global states of distributed systems. Transactions on Computer Systems 3(1), 63-75 (1985)

7. Dongarra, J., Beckman, P., et al.: The international exascale software roadmap. Intl. Journal of High Performance Computer Applications 25(11) (to appear) (2011)

8. Esteban Meneses, C.L.M., Kalé, L.V.: Team-based message logging: Preliminary results. In: 3rd Workshop on Resiliency in High Performance Computing (Resilience) in Clusters, Clouds, and Grids (CCGRID 2010) (May 2010)

9. Gabriel, E., Fagg, G.E., Bosilca, G., Angskun, T., Dongarra, J.J., Squyres, J.M., Sahay, V., Kambadur, P., Barrett, B., Lumsdaine, A., Castain, R.H., Daniel, D.J., Graham, R.L., Woodall, T.S.: Open MPI: Goals, concept, and design of a next generation MPI implementation. In: Proceedings, 11th European PVM/MPI Users' Group Meeting, Budapest, Hungary, pp. 97-104 (September 2004)

10. Gao, Q., Huang, W., Koop, M.J., Panda, D.K.: Group-based coordinated checkpointing for mpi: A case study on infiniband. In: International Conference on Parallel Processing, ICPP 2007 (2007)

11. Ho, J.C.Y., Wang, C.L., Lau, F.C.M.: Scalable Group-based Checkpoint/Restart for Large-Scale Message-Passing Systems. In: Proceedings of the 22nd IEEE International Symposium on Parallel and Distributed Processing (IPDPS), pp. 1-12. IEEE, Los Alamitos (2008)

12. Hlary, J.M., Mostefaoui, A., Raynal, M.: Communication-induced determination of consistent snapshots. IEEE Transactions on Parallel and Distributed Systems 10(9), 865-877 (1999)

13. Kale, L.: Charm++. In: Padua, D. (ed.) Encyclopedia of Parallel Computing, Springer, Heidelberg (to appear) (2011)

14. Lamport, L.: Time, clocks, and the ordering of events in a distributed system. Communications of the ACM 21(7), 558-565 (1978)

15. Lemarinier, P., Bouteiller, A., Herault, T., Krawezik, G., Cappello, F.: Improved message logging versus improved coordinated checkpointing for fault tolerant MPI. In: IEEE International Conference on Cluster Computing. IEEE CS Press, Los Alamitos (2004)

16. Negara, S., Pan, K.C., Zheng, G., Negara, N., Johnson, R.E., Kale, L.V., Ricker, P.M.: Automatic MPI to AMPI Program Transformation. Tech. Rep. 10-09, Parallel Programming Laboratory (March 2010)

17. Plank, J.S.: Efficient Checkpointing on MIMD Architectures. Ph.D. thesis, Princeton University (June 1993), http://www.cs.utk.edu/ plank/plank/papers/thesis.html

18. Rao, S., Alvisi, L., Vin, H.M.: The cost of recovery in message logging protocols. In: 17th Symposium on Reliable Distributed Systems (SRDS), October 1998, pp. 10-18. IEEE CS Press, Los Alamitos (1998)

19. The MPI Forum: MPI: a message passing interface. In: Supercomputing 1993: Proceedings of the 1993 ACM/IEEE Conference on Supercomputing, pp. 878-883. ACM Press, New York (1993) 